\title{
Netzwerk für optimale Therapie bei schwerem allergischen Asthma
}

\begin{abstract}
S chweres allergisches Asthma ist diagnostisch und therapeutisch eine komplexe Herausforderung", erklärte Prof. Karl-Christian Bergmann aus Berlin. Trotz intensiver Therapie ist die Asthmasymptomatik bei diesen Patienten nicht zu kontrollieren. Auch die orale Steroidtherapie hilft häufig nicht weiter. Es wird geschätzt, dass etwa 100.000 Menschen in Deutschland unter schwerem Asthma leiden und dass das Asthma bei jedem Zweiten allergischer Genese ist.

Das „Netzwerk Schweres Allergisches Asthma (SAA)"soll helfen, die Situation dieser Patienten zu verbessern und den niedergelassenen Arzt bei der Diagnose und Therapie der Erkrankung zu unterstützen. Es wurde im April 2008 für den Bereich Berlin/Brandenburg
\end{abstract}

vom Allergie-Centrum-Charité gegründet und wird durch die Novartis Pharma $\mathrm{GmbH}$ unterstützt. Konzept und Struktur des Netzwerks erläutert Bergmann so: „Der behandelnde Arzt überweist schwierig oder nur unzureichend therapierbare Patienten an das klinische Netzwerk-Zentrum. Das Zentrum schlägt Behandlungsstrategien vor, die auch die Therapie mit Anti-IgE-Antikörpern einschließen können, und rezeptiert gegebenenfalls die Medikamente. Die Therapie selbst übernimmt der Niedergelassene, mit dem wir in der Folge individuelle Krankheitsverläufe einzelner Patienten im kollegialen Gespräch gemeinsam auswerten."

Ansprechpartner im Netzwerk sind Prof. Dr. Karl-Christian Bergmann (Allergie-Centrum-Charité) und Prof. Dr.

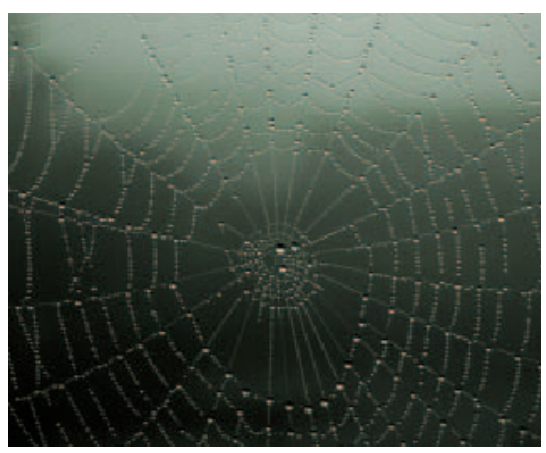

Vernetzen hilft, Patienten mit schwerem Asthma adäquat zu versorgen.

Eckard Hamelmann (Ruhr-Universität Bochum), der für Kinder und Jugendliche zuständig ist.

$k o c$

Pressekonferenz „Gemeinsam für den Patienten handeln - das neue Netzwerk Schweres Allergisches Asthma (SAA)". Berlin, 14. April 2008. Veranstalter: Novartis Pharma, Nürnberg

\section{Innovationspreis für Gräser-Impf-Tablette}

A m 5. April 2008 erhielt ALKSCHERAX den diesjährigen H. G. Creutzfeldt-Innovationspreis für die Gräser-Impf-Tablette GRAZAX ${ }^{\circledR}$. Das H. G. Creutzfeldt-Institut legte bei der Vergabe des Preises hohe Maßstäbe an:

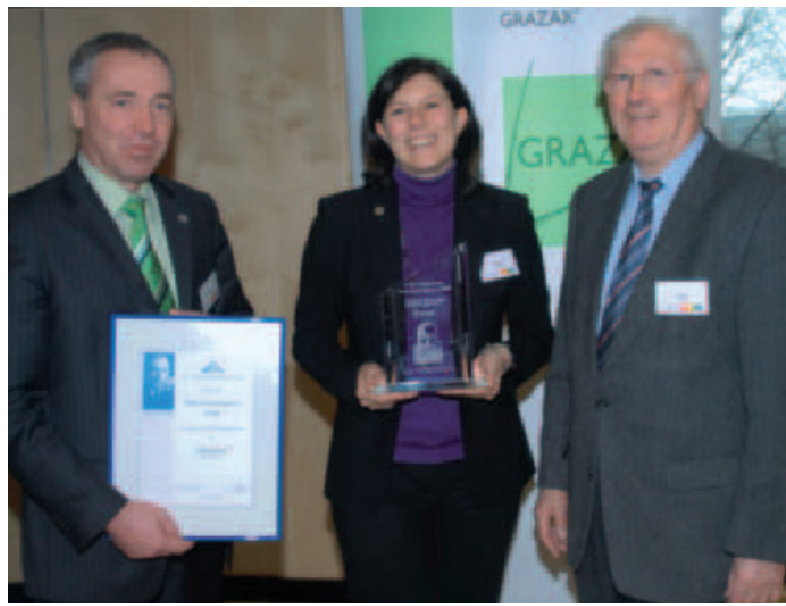

Dr. Eckhard Puchert (links), Geschäftsführer von ALKSCHERAX Arzneimittel GmbH, und Konstanze Fleige (Mitte), Produktmanagerin von GRAZAX $^{\circledR}$, nahmen den Preis von Prof. Dr. Dieter Müller vom H.-G.-Creutzfeld-Institut zu Kiel entgegen.
Das auszuzeichnende Arzneimittel musste innovativ und in kontrollierten Studien überzeugend dokumentiert sein, kausal immunologisch wirken, den Krankheitsverlauf bei hoher klinischer Sicherheit und Verträglichkeit verbessern, die Alltagskompetenz erhalten und die Lebensqualität steigern. „Die Entscheidung fiel der Jury in diesem Jahr nicht schwer", sagte Prof. Dr. Wolf-Dieter Gerber vom H. G. Creutzfeldt-Institut zu Kiel. „GRAZAX ${ }^{\circledR}$ ist als erstes zugelassenes Präparat für die einfache und sichere $\mathrm{Ta}$ blettenimmuntherapie zu Hause eine echte Innovation in der kausalen Allergietherapie." Die Gräser-Impf-Tablette enthält gefriergetrocknete Allergene aus Gräserpollen. Un- ter die Zunge gelegt, löst sich die Tablette sekundenschnell auf, so dass die freigesetzten Allergene von der Mundschleimhaut aufgenommen werden können. Dort wirken sie mithilfe antigenpräsentierender Zellen auf T-Lymphozyten und induzieren eine immunologische Toleranz gegenüber Allergenen aus eingeatmeten Gräser- und Roggenpollen. Die Anwendung wird über drei Jahre empfohlen. Das Entwicklungsprogramm hat die klinische Wirksamkeit und die Sicherheit der Therapie an über 2.400 Patienten bestätigt. Mit fortschreitender Therapiedauer wurde eine zunehmende Wirkung beobachtet. „Mit hoher Wahrscheinlichkeit hat die Gräser-Impf-Tablette ebenso wie die subkutan verabreichte Immuntherapie mit ALK-SQ depot oder Allergovit eine nachgewiesene sehr gute Wirksamkeit bei Gräser- und Roggenpollenallergie, die bei einer Therapie über drei Jahre zunimmt", sagte Professor Dr. Gerd Rasp aus Salzburg anlässlich der Preisverleihung.

Nach einer Pressemitteilung von ALKSCHERAX, Wedel 